\title{
Hip Adductor Longus Tendon Origin Anatomy Is Consistent and May Inform Surgical Reattachment
}

\author{
Michael A. Perrone, M.D., M.P.H., Ali Noorzad, M.D., Mathew Hamula, M.D., \\ Melodie Metzger, Ph.D., Michael Banffy, M.D., and Michael Gerhardt, M.D.
}

\begin{abstract}
Purpose: To define the topographic anatomy of the footprint of the adductor longus origin on the pubis and its underlying bony morphology to better inform surgical repair of adductor longus tendon injuries. Methods: Five cadaveric pelvis specimens were dissected, making 10 adductor footprints available for analysis. The adductor longus tendon origin was isolated and the surrounding tissue debrided. The circumference of the tendinous attachment to the pubic crest was marked before excising the tendon and fibrocartilage enthesis from the pubis. Radiopaque paint was prepared by mixing $30 \mathrm{~mL}$ of all-purpose acrylic paint (Anita's no. 11150 Island Blue; Rust-Oleum Corp, Vernon Hills, IL) with 15g of E-Z-HD 98\% w/w barium sulfate (Bracco Diagnostics Inc., Anjou Quebec, Canada) and applied to the marked footprint. The specimens underwent a 1.0-mm slice computed tomographic scan with 3-dimensional reconstructions. Synapse PACS (FujiFilm, Valhalla, NY) software for measurements of the tendon footprint and underlying bone. Results: Average age and weight of the specimens at the time of death was 37 years and $204.6 \pm 48.7 \mathrm{lbs}$, respectively. The width and length of the tendon origin was $12.0 \pm 1.1 \mathrm{~mm}$ and $10.9 \pm 1.1 \mathrm{~mm}$, respectively. The distance of the center of the footprint from the center of the pubic tubercle was $8.5 \pm 1.4 \mathrm{~mm}$ lateral and $12.2 \pm 0.4 \mathrm{~mm}$ caudal. The osseous thickness underlying the footprint was $18.7 \pm 3.7 \mathrm{~mm}$ at an angle of $34.5 \pm 1.5^{\circ}$ in relation to the sagittal plane. The correlation between specimen body weight and the thickness of the bone underlying the footprint was strongly positive $(r=0.92)$. Conclusions: We found that there is a consistent angle from the center of the adductor longus tendon footprint to the point of maximal underlying bony thickness, as well as a positive correlation between body mass index and osseous thickness, which may inform anatomic reattachment of this tendon. Clinical Relevance: Our findings will assist surgeons in identifying the footprint of the adductor longus tendon and safely perform anatomic repair of adductor longus tendon avulsions.
\end{abstract}

From Cedars Sinai Kerlan-Jobe Orthopaedic Institute (M.A.P., M.H., M.B., M.G.); Cedars Sinai Department of Orthopaedics (A.N.); The Metzger Biomechanics Laboratory at Cedars-Sinai Medical Center (M.M.); and Santa Monica Orthopaedic and Sports Medicine Group (M.G.), Los Angeles, California, U.S.A.

The authors report the following potential conflicts of interest or sources of funding: M.G. reports personal fees and nonfinancial support from Arthrex, personal fees and nonfinancial support from Exactech, personal fees and nonfinancial support from Medical Device Business Services, personal fees and nonfinancial support from Medacta USA, personal fees and nonfinancial support from Stryker, personal fees and nonfinancial support from DePuy Synthes, and personal fees and nonfinancial support from Anika Therapeutics, outside the submitted work. M.B. reports personal fees and nonfinancial support from Stryker Endoscopy, personal fees and nonfinancial support from Smith $\theta$ Nephew, and personal fees and nonfinancial support from Vericel, outside the submitted work. Full ICMJE author disclosure forms are available for this article online, as supplementary material.

Received June 22, 2020; accepted September 19, 2020

Address correspondence to Michael A. Perrone, M.D., M.P.H., Cedars Sinai Kerlan-Jobe Orthopaedic Institute, 6801 Park Terrace, Suite 400, Los Angeles, CA 90045.E-mail: perronearthroscopy@gmail.com

(C) 2020 THE AUTHORS. Published by Elsevier Inc. on behalf of the Arthroscopy Association of North America. This is an open access article under the CC BY-NC-ND license (http://creativecommons.org/licenses/by-nc-nd/4.0/).

2666-061X/20988

https://doi.org/10.1016/j.asmr.2020.09.015
S ports-related groin injuries are common among athletes. They can be seen in a variety of sports and occur most frequently through noncontact mechanisms when the lower extremity experiences eccentric loading with forced abduction, hip extension, and external rotation. ${ }^{1,2}$ In one study of professional soccer players, $64 \%$ of groin injuries resulted in damage to a portion of the adductor muscle complex, with the adductor longus being the most commonly injured structure. ${ }^{3}$ These injuries can present either acutely, characterized by an audible pop, groin pain and ecchymosis, or chronically as dull discomfort in the groin exacerbated by activity.

Although diagnosis begins with a thorough history and physical examination, ${ }^{4}$ these injuries are usually confirmed with advanced imaging modalities like ultrasound or magnetic resonance imaging (MRI). ${ }^{5,6} \mathrm{~A}$ recent MRI study on acute groin injuries confirmed that the adductor longus muscle is the most frequently injured structure and reported that $26 \%$ of these injuries occurred at the proximal insertion on the pubis. ${ }^{7}$ 


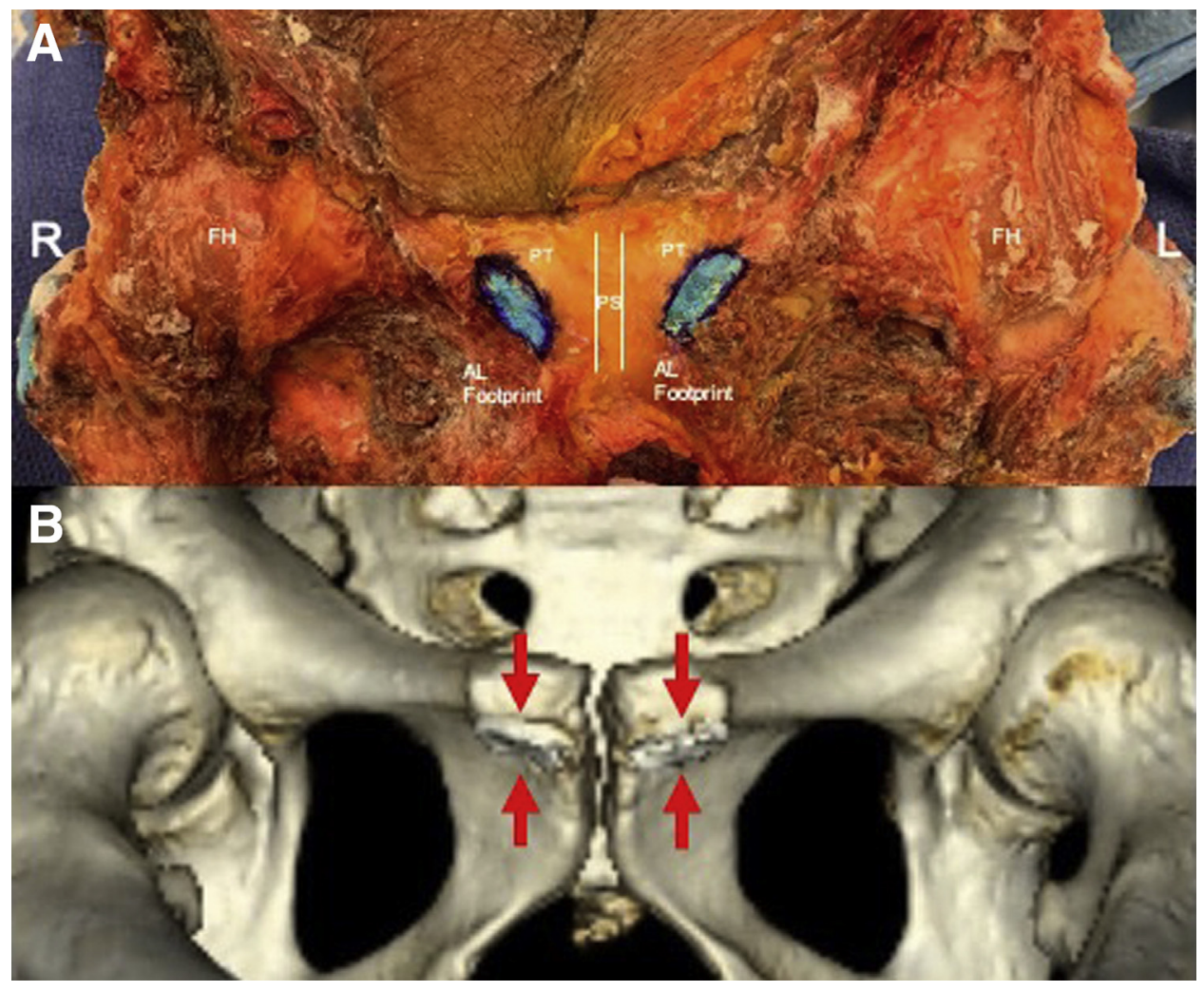

Fig 1. A, Specimen following dissection with adductor longus footprints marked with radiopaque paint. B, 3-Dimensional computed tomography reconstruction of the pelvis specimen with radiopaque markings visible (arrows). (AL, adductor longus; FH, femoral Head; PS, pubic symphysis; PT, pubic tubercle.)

As this injury has become increasingly more recognized through greater understanding of injury patterns and improvements in diagnostic imaging modalities, ${ }^{8-11}$ surgical repair of proximal avulsions has become more common. ${ }^{12,13}$ Multiple case series have reported excellent outcomes and near-full recovery in athletic populations; however, this remains a controversial topic, with advocates for both operative and nonoperative management. ${ }^{13-20}$

Anatomic reattachment is the goal of operative intervention, and several studies have described the anatomic features of the origin of the adductor longus tendon to inform surgical repairs. ${ }^{21-24}$ The purpose of this study is to define the topographic anatomy of the footprint of the adductor longus origin on the pubis and its underlying bony morphology to better inform surgical repair of adductor longus tendon injuries. We hypothesize that there will be a consistent pubic morphology that can inform anchor placement in the trajectory that provides the greatest bony purchase.

\section{Methods}

Dissection of the adductor longus tendon origin in 5 cadaveric pelvises was performed. All specimens dissected included the pelvis to mid-diaphyseal femur. The tendon origin was isolated with blunt dissection. The tissue around the adductor tendon origin was sharply debrided to better isolate the tendon. The circumference of the tendinous attachment to the pubic crest was outlined with a marking pen. The tendon along with its fibrocartilage enthesis was then sharply dissected from its origin on the pubis. Any remaining tissue within the markings was sharply detached and curetted from the anterior pubis, caudal to the pubic crest.

Radiopaque paint was prepared by mixing $30 \mathrm{~mL}$ of all-purpose acrylic paint (Anita's no. 11150 Island Blue; Rust-Oleum Corp, Vernon Hills, IL) with $15 \mathrm{~g}$ of E-ZHD 98\% w/w barium sulfate (Bracco Diagnostics Inc., Anjou Quebec, Canada). Two layers of radiopaque paint were then applied to the marked area, with time allowed for each layer to dry (Fig 1A). The specimens underwent a 1.0-mm slice computed tomographic (CT) scan with 3-dimensional reconstructions. Synapse PACS (FujiFilm, Valhalla, NY) software was used to perform measurements of the tendon footprint as well as the underlying bone (Fig 1B).

The dimensions of the tendon were measured at the largest section of the tendon in each plane. The sagittal plane was used to measure tendon length and the axial plane to measure width. The distance of the center of the tendon origin to center of the pubic tubercle was also measured.

The thickness of the bone underlying the footprint was measured in the sagittal plane from the bony footprint to the far cortex in a plane that provided the 


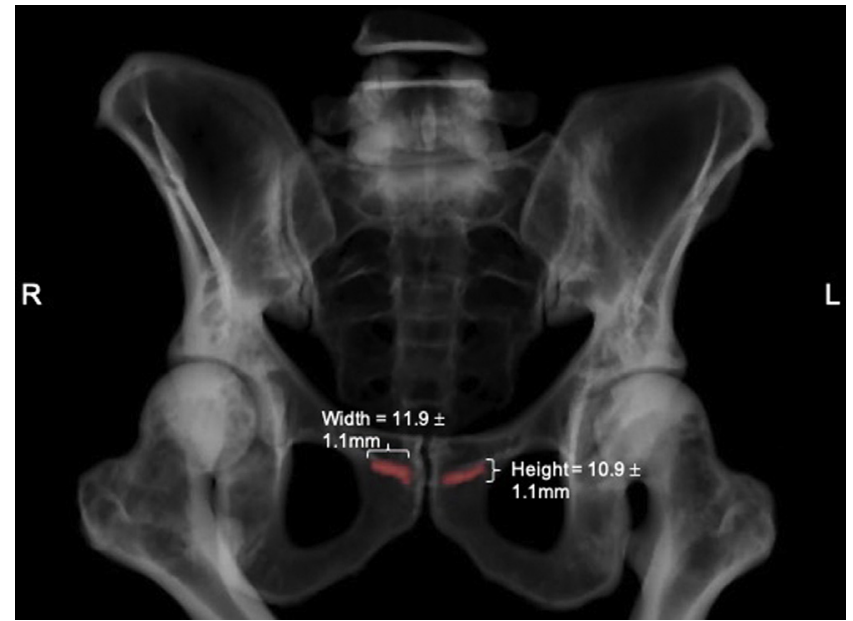

Fig 2. "Ghost" tomographic reconstruction of the pelvis specimen with radiopaque markings highlighted.

maximum thickness. This angle was then measured in reference to the plane parallel to the table with the pelvis in the supine position (Fig 2B).

Data are presented as means with standard deviations. Pearson correlation was used to measure associations between continuous variables.

This study did not represent research with living individuals or the use of protected health information and was declared exempt by our institutional review board.

\section{Results}

Five male pelvis specimens were dissected for a total of 10 adductor tendons. No specimen had gross evidence of previous pelvic surgery, though one had previous L3-L5 instrumented spinal fusion. The average age of the specimens at the time of death was 37 years (28-47 years). Average weight was $204.6 \pm 48.7 \mathrm{lbs}$ (Table 1). The mean width of the tendon origin was $12.0 \pm 1.1 \mathrm{~mm}$ and the mean length was $10.9 \pm 1.1$ mm (Fig 2). The mean distance from the center of the pubic tubercle to the center of the tendon origin was 8.5 $\pm 1.4 \mathrm{~mm}$ lateral and $12.2 \pm 0.4 \mathrm{~mm}$ caudal. The mean osseous thickness underlying the footprint was $18.7 \pm$ $3.7 \mathrm{~mm}$ (Fig 3A). The angle subtended by the center of the adductor tendon origin to the far cortex of the pubis with maximum thickness in relation to the horizontal plane was $34.5 \pm 1.5^{\circ}$ (Fig 3B). There was a strongly positive correlation $(r=0.92)$ between the body weight of the specimen and the thickness of the bone underlying the footprint (Fig 4).

\section{Discussion}

Our study describes the topographic anatomy of the adductor longus tendon footprint on the pubis, demonstrating a consistent morphology of the underlying bony thickness in relation to the footprint, as well as a positive correlation between patient body mass index and bony thickness.

The use of suture anchors at the adductor longus footprint is the most often reported method of repair. Common anchor systems employ either a drill or punch to create pilot holes in the pubis for insertion of the anchor(s). Care must be taken not to penetrate too deeply through the far cortex and into the space of Retzius, where there is the potential for injury to the bladder. While a commercially available anchor may

Table 1. Measurement of the Dimensions of the Adductor Longus Footprint per Specimen

\begin{tabular}{|c|c|c|c|c|c|c|}
\hline Specimen & $\# 1$ & $\# 2$ & \#3 & $\# 4$ & \#5 & Mean (SD) \\
\hline Age at death, y & 47 & 46 & 41 & 28 & 23 & 37 \\
\hline Weight, lbs & 178 & 140 & 250 & 255 & 200 & $204.6(48.7)$ \\
\hline \multicolumn{7}{|l|}{ Footprint width, mm } \\
\hline $\mathrm{L}$ & 11.8 & 9.8 & 12.4 & 13.2 & 12.4 & $12(1.1)$ \\
\hline $\mathrm{R}$ & 11.3 & 10.6 & 12.9 & 13.1 & 12.2 & \\
\hline \multicolumn{7}{|l|}{ Footprint height, mm } \\
\hline $\mathrm{L}$ & 10.3 & 10.5 & 12.3 & 12.3 & 9.9 & $10.9(1.1)$ \\
\hline $\mathrm{R}$ & 9.6 & 9.8 & 11.6 & 12.0 & 10.3 & \\
\hline \multicolumn{7}{|c|}{ Lateral distance from Pubic tubercle, mm } \\
\hline $\mathrm{L}$ & 7.0 & 6.0 & 9.0 & 10.0 & 9.0 & $8.5(1.4)$ \\
\hline $\mathrm{R}$ & 8.0 & 7.0 & 9.0 & 10.0 & 10.0 & \\
\hline \multicolumn{7}{|c|}{ Caudal distance from pubic tubercle, $\mathrm{mm}$} \\
\hline $\mathrm{L}$ & 12.0 & 12.0 & 12.0 & 13.0 & 12.0 & $12.2(0.4)$ \\
\hline $\mathrm{R}$ & 12.0 & 12.0 & 12.0 & 13.0 & 12.0 & \\
\hline \multicolumn{7}{|l|}{ Osseous thickness, mm } \\
\hline $\mathrm{L}$ & 16.1 & 14.5 & 19.9 & 25.2 & 18.2 & $18.7(3.7)$ \\
\hline $\mathrm{R}$ & 16.0 & 14.7 & 20.6 & 24.1 & 17.4 & \\
\hline \multicolumn{7}{|c|}{ Angle of maximum thickness } \\
\hline $\mathrm{L}$ & $37^{\circ}$ & $34^{\circ}$ & $35^{\circ}$ & $35^{\circ}$ & $35^{\circ}$ & $34.5^{\circ}(1.5)$ \\
\hline $\mathrm{R}$ & $33^{\circ}$ & $35^{\circ}$ & $33^{\circ}$ & $32^{\circ}$ & $36^{\circ}$ & \\
\hline
\end{tabular}

L, left; R, right; SD, standard deviation. 


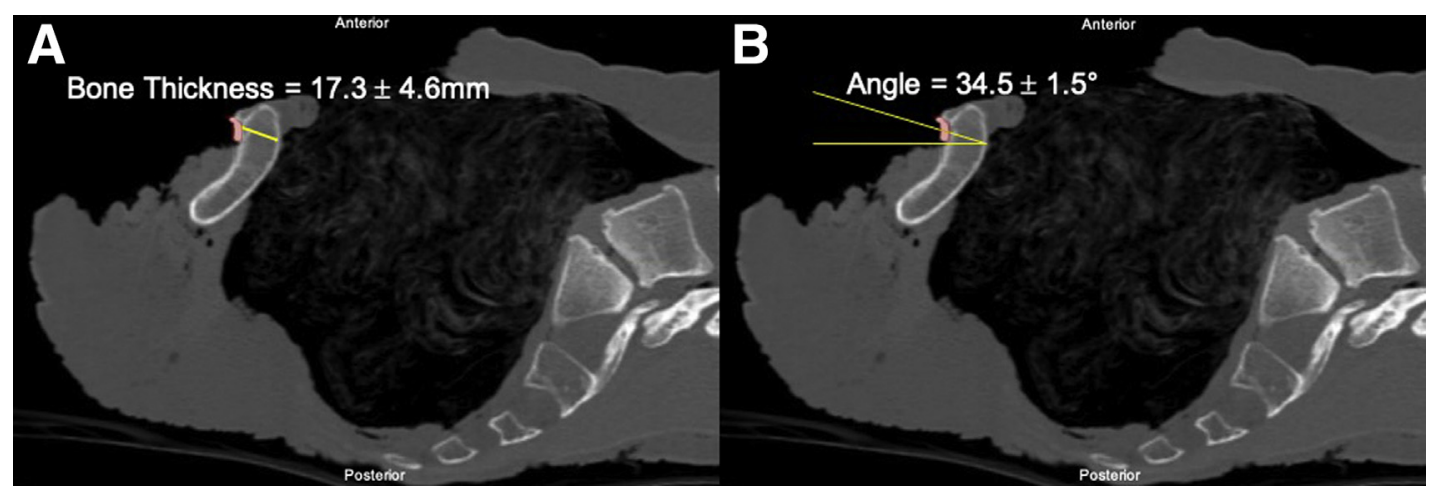

Fig 3. Sagittal plane computed tomography scan of pelvis specimen with radiopaque marking highlighted. (A) Measurement of bony thickness underlying footprint. (B) Angle from bony print to area of maximum bony thickness.

have an effective length of $14 \mathrm{~mm}$, drilling or punch devices can extend to a depth of 2 to $4 \mathrm{~mm}$ beyond the length of the anchor. ${ }^{25}$ The bone underlying the footprint had a maximum thickness $18.7 \pm 3.7 \mathrm{~mm}$ at an angle of $34.5 \pm 1.5^{\circ}$ from the footprint in relation to the horizontal plane and was positively correlated with patient body weight.

The adductor longus tendon originates from the inferior part of the pubic crest and contains a fibrocartilaginous enthesis. This footprint lies approximately $8.5 \mathrm{~mm}$ lateral and $12.2 \mathrm{~mm}$ caudal to the pubic tubercle, according to our results. Riff et al. ${ }^{22}$ reported that the adductor longus tendon origin lies $1.5 \mathrm{~cm}$ from the adductor brevis and $0.69 \mathrm{~cm}$ from the rectus abdominis, on average, with a high degree of variability. Our study instead chose the pubic tubercle as the reference point for measurements, as many surgeons rely on tactile feedback from bony prominences to localize the footprint and guide their repairs.

Strauss et al. ${ }^{24}$ reported that the average length and width of the tendon at the origin was $11.6 \mathrm{~mm}$ and 3.7 $\mathrm{mm}$, respectively. Our study found an average length of $10.9 \mathrm{~mm}$ and width of $12 \mathrm{~mm}$; however, our measurements included the underlying fibrocartilaginous enthesis. It has been the senior surgeon's observation, which has been corroborated in the literature, that tendinous avulsions can have remnants of fibrocartilage on the stump. ${ }^{14}$ Consideration of this enthesis and its role in the attachment point of the adductor longus may lead to a more anatomic repair.

During the approach for adductor longus repair, it has been observed that the fascia overlying the tendon is often intact. ${ }^{20}$ Schilders et al., ${ }^{21}$ in a cadaveric analysis provided evidence to this observation by describing an end-to-end connection between the adductor longus tendon and the pyramidalis muscle through the anterior pubic ligament. They called this the pyramidalis-anterior pubic-ligament-adductor longus complex, and it may be implicated in other core muscle injuries. ${ }^{21}$ Upon closer inspection under the tendon, a separation of the underlying tendon and fibrocartilage from the pubis is usually observed in

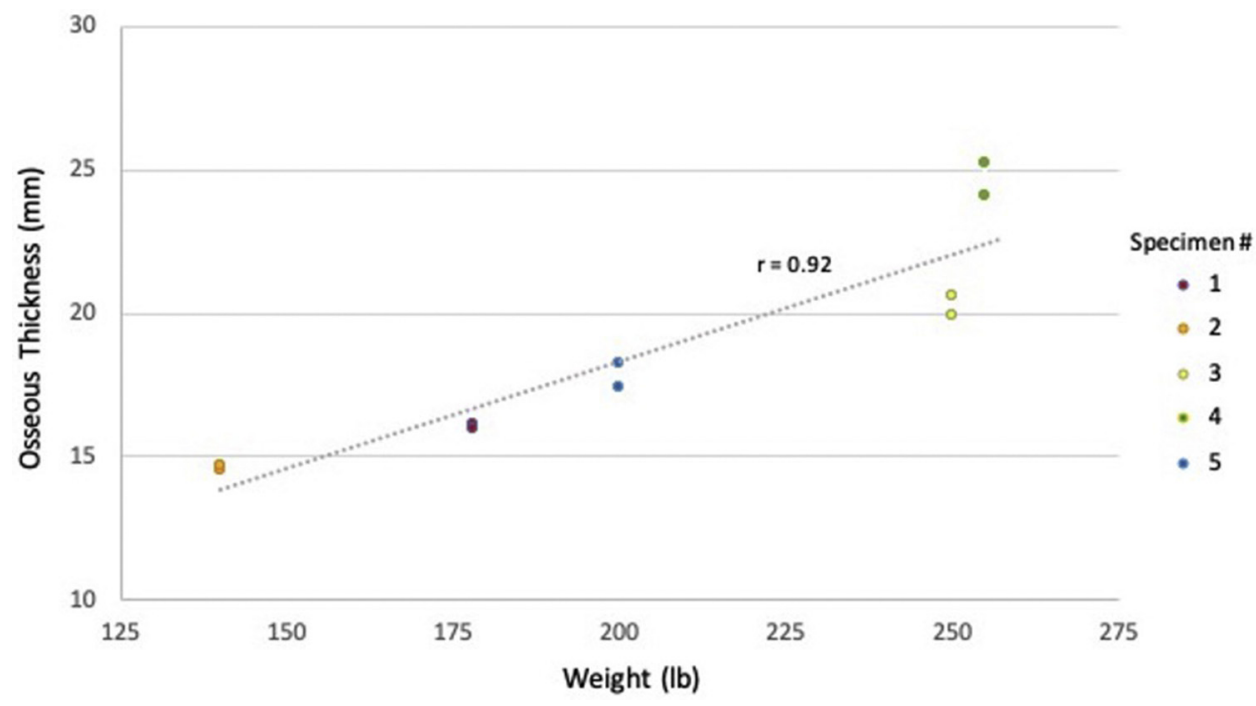

Fig 4. Correlation of specimen body weight (lb) and maximum osseous thickness underlying adductor longus footprint. 
patients undergoing repair. Further anatomic variation may exist at the footprint, as Davis et al. ${ }^{26}$ discovered, where a fusion between the adductor longus and gracilis tendons was noted in $10 \%$ of specimens. We did not observe this anatomic variation in any of our specimens.

These findings highlight the importance of considering each patients' unique anatomy before performing an adductor longus repair. Improved knowledge of the anatomic footprint of the adductor longus tendon reduces the risk of a nonanatomic repair, potentially translating into a more physiologic kinematics, and in turn, a more rapid recovery and expedited return to training and play.

\section{Limitations}

This study is not without limitations. Using barium paint and a CT scanner to image tendon footprint topography is a well-described technique, although it is not without its disadvantages. ${ }^{27,28}$ The accuracy of this technique depends on how accurately the footprints are defined by dissection and subsequently hand painted. Intra- and interobserver reliability were not calculated, since outlining and painting the tendon insertion by hand could only be performed once. Other techniques rely on similar potential sources of human error in that they involve outlining ligament footprints by hand before imaging. ${ }^{29,30}$ Despite the accuracy of the CT scanner, measuring the tendon dimensions, bony thickness, and angle of maximum bony thickness was ultimately up to the discretion of the investigator and a potential source of error. Other limitations of our study include the possibility of a spectrum of anatomic variation that was not seen given our small sample size. These measurements may not be generalizable to females as all of the specimens in our analysis were males, though the majority of adductor longus injuries occur in male athletes. ${ }^{31}$ However, we did use a female pelvis specimen as a control to hone our technique and methods. While this specimen was considerably smaller (90 lbs) than the male specimens, with smaller tendon dimensions and osseous thickness, the angle of maximum osseous thickness was $36^{\circ}$, suggesting some degree of consistent morphology between genders in this particular region of the pelvis.

\section{Conclusions}

This study provides a topographic description of adductor longus origin footprint and the underlying bony morphology. The hypothesis that there would be a consistent pubic morphology in relation to the adductor longus footprint was supported. There was a positive correlation between the patient's body mass index and the thickness of the pubis as well as a consistent angle from the center of the footprint to the point of maximal bony thickness that may assist surgeons in performing anatomic reattachment of this tendon.

\section{Acknowledgments}

The authors acknowledge Francisco Huizar and John Hale of Arthrex Education. While Arthrex Education provided no funding for this project, they made their on-site surgical skills laboratory at Kerlan-Jobe available for storage and dissection of the specimens. We would also like to acknowledge Julien Legay, CT Technologist at Kerlan-Jobe Orthopaedic Institute, for facilitating the CT scanning of the specimens and providing the 3-dimensional reconstructions used for our figures.

\section{References}

1. Serner A, Mosler AB, Tol JL, Bahr R, Weir A. Mechanisms of acute adductor longus injuries in male football players: A systematic visual video analysis. Br J Sports Med 2019;53:158-164.

2. Ueblacker P, English B, Mueller-Wohlfahrt HW. Nonoperative treatment and return to play after complete proximal adductor avulsion in high-performance athletes. Knee Surg Sports Traumatol Arthrosc 2016;24:3927-3933.

3. Werner J, Hägglund M, Waldén M, Ekstrand J. UEFA injury study: A prospective study of hip and groin injuries in professional football over seven consecutive seasons. $\mathrm{Br}$ J Sports Med 2009;43:1036-1040.

4. Suarez JC, Ely EE, Mutnal AB, et al. Comprehensive approach to the evaluation of groin pain. J Am Acad Orthop Surg 2013;21:558-570.

5. Omar IM, Zoga AC, Kavanagh EC, et al. Athletic pubalgia and "sports hernia": Optimal MR imaging technique and findings. Radiographics 2008;28:1415-1438.

6. Robinson P, Barron DA, Parsons W, Grainger AJ, Schilders EM, O'Connor PJ. Adductor-related groin pain in athletes: Correlation of MR imaging with clinical findings. Skeletal Radiol 2004;33:451-457.

7. Serner A, Weir A, Tol JL, et al. Characteristics of acute groin injuries in the adductor muscles: A detailed MRI study in athletes. Scand J Med Sci Sports 2018;28:667-676.

8. Elattar O, Choi HR, Dills VD, Busconi B. Groin Injuries (athletic pubalgia) and return to play. Sports Health 2016;8:313-323.

9. Weir A, Brukner P, Delahunt E, et al. Doha agreement meeting on terminology and definitions in groin pain in athletes. Br J Sports Med 2015;49:768-774.

10. Palisch A, Zoga AC, Meyers WC. Imaging of athletic pubalgia and core muscle injuries: clinical and therapeutic correlations. Clin Sports Med 2013;32:427-447.

11. Zoga AC, Kavanagh EC, Omar IM, et al. Athletic pubalgia and the "sports hernia": MR imaging findings. Radiology 2008;247:797-807.

12. Muschaweck U, Berger L. Minimal repair technique of sportsmen's groin: An innovative open-suture repair to treat chronic inguinal pain. Hernia 2010;14:27-33.

13. Bharam S, Feghhi DP, Porter DA, Bhagat PV. Proximal adductor avulsion injuries: Outcomes of surgical 
reattachment in athletes. Orthop J Sports Med 2018;6: 2325967118784898.

14. Dimitrakopoulou A, Schilders EM, Talbot JC, Bismil Q. Acute avulsion of the fibrocartilage origin of the adductor longus in professional soccer players: A report of two cases. Clin J Sport Med 2008;18:167-169.

15. Schlegel TF, Bushnell BD, Godfrey J, Boublik M. Success of nonoperative management of adductor longus tendon ruptures in National Football League athletes. Am J Sports Med 2009;37:1394-1399.

16. Vogt S, Ansah P, Imhoff AB. Complete osseous avulsion of the adductor longus muscle: Acute repair with three fiberwire suture anchors. Arch Orthop Trauma Surg 2007;127:613-615.

17. Rizio L, Salvo JP, Schürhoff MR, Uribe JW. Adductor longus rupture in professional football players: Acute repair with suture anchors: a report of two cases. Am J Sports Med 2004;32:243-245.

18. Tansey RJ, Benjamin-Laing $H$, Jassim S, Liekens $K$, Shankar A, Haddad FS. Successful return to high-level sports following early surgical repair of combined adductor complex and rectus abdominis avulsion. Bone Joint J 2015;97-B:1488-1492.

19. Verrall GM, Slavotinek JP, Fon GT, Barnes PG. Outcome of conservative management of athletic chronic groin injury diagnosed as pubic bone stress injury. Am J Sports Med 2007;35:467-474.

20. Gerhardt M, Sherman B, Trentacosta N, Hobart S, Hutchinson W, Chahla J. Adductor tendon repair: Case report and description of a novel approach for improved exposure. Tech Orthop.2019;1

21. Schilders E, Bharam S, Golan E, et al. The pyramidalisanterior pubic ligament-adductor longus complex (PLAC) and its role with adductor injuries: A new anatomical concept. Knee Surg Sports Traumatol Arthrosc 2017:25:3969-3977.
22. Riff AJ, Movassaghi K, Beck EC, et al. Surface mapping of the musculotendinous attachments at the pubic symphysis in cadaveric specimens: Implications for the treatment of core muscle injury. Arthroscopy 2019;35: 2358-2364.

23. Tuite DJ, Finegan PJ, Saliaris AP, Renström PA, Donne B, O'Brien M. Anatomy of the proximal musculotendinous junction of the adductor longus muscle. Knee Surg Sports Traumatol Arthrosc 1998;6:134-137.

24. Strauss EJ, Campbell K, Bosco JA. Analysis of the crosssectional area of the adductor longus tendon: A descriptive anatomic study. Am J Sports Med 2007;35:996-999.

25. Arthrex Inc. The Fully Threaded Family of Soft Tissue Anchor Repairs. Acchttps://www.arthrex.com/resources/ brochures/sjjhY_kEEeCRTQBQVoRHOw/the-fullythreaded-family-of-soft-tissue-repair-anchors. essed May 31, 2020.

26. Davis JA, Stringer MD, Woodley SJ. New insights into the proximal tendons of adductor longus, adductor brevis and gracilis. Br J Sports Med 2012;46:871-876.

27. Neuschwander TB, Indresano AA, Hughes TH, Smith BW. Footprint of the lateral ligament complex of the ankle. Foot Ankle Int 2013;34:582-586.

28. Neuschwander TB, Benke MT, Gerhardt MB. Anatomic description of the origin of the proximal hamstring. Arthroscopy 2015;31:1518-1521.

29. Forsythe B, Harner C, Martins CA, Shen W, Lopes OV, Fu FH. Topography of the femoral attachment of the posterior cruciate ligament. Surgical technique. J Bone Joint Surg Am 2009;91:89-100 (suppl 2).

30. Lopes OV, Ferretti M, Shen W, Ekdahl M, Smolinski P, Fu FH. Topography of the femoral attachment of the posterior cruciate ligament. J Bone Joint Surg Am 2008;90: 249-255.

31. Orchard JW. Men at higher risk of groin injuries in elite team sports: A systematic review. Br J Sports Med 2015;49:798-802. 